\title{
Peindre la voix, écrire le déchet
}

Painting Voice, Writing Waste

\section{Agnès Jeanjean}

\section{OpenEdition}

Journals

Édition électronique

URL : https://journals.openedition.org/tc/8075

DOI : $10.4000 /$ tc. 8075

ISSN : 1952-420X

\section{Éditeur}

Éditions de l'EHESS

\section{Édition imprimée}

Date de publication : 31 octobre 2016

Pagination : 310-321

ISBN : 9782713225291

ISSN : 0248-6016

\section{Référence électronique}

Agnès Jeanjean, «Peindre la voix, écrire le déchet », Techniques \& Culture [En ligne], 65-66 | 2016, mis en ligne le 31 octobre 2018, consulté le 29 septembre 2022. URL : http://journals.openedition.org/tc/ 8075 ; DOl : https://doi.org/10.4000/tc. 8075 


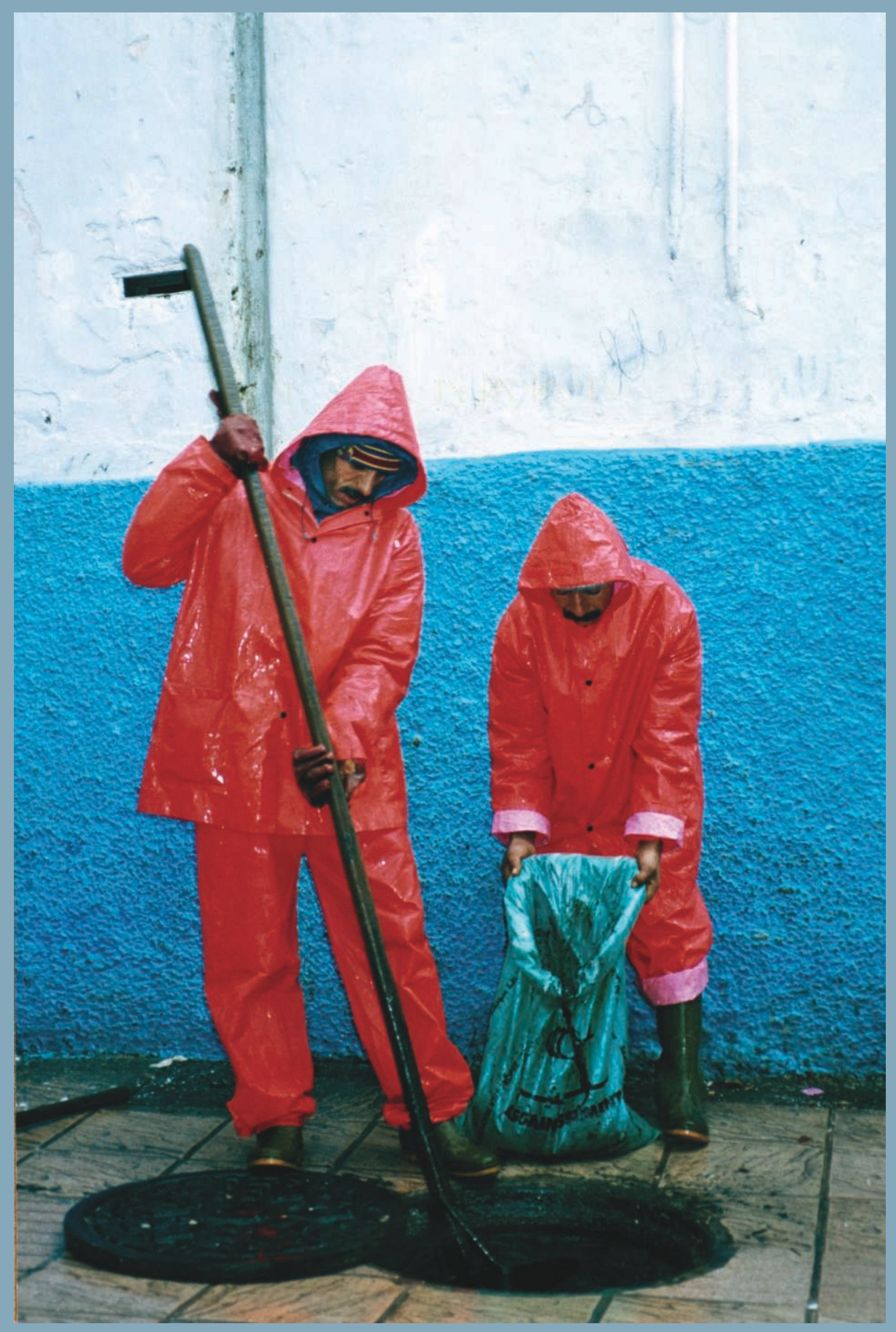




\section{Peindre la voix, écrire le déchet}

\section{Les déchets ne utricheraient pas "}

Pour qui s'intéresse aux déchets, il est des citations incontournables. L'ordure y est envisagée comme une voie d'accès à la vérité. Il en va ainsi des passages que Victor Hugo (1862) a consacrés, dans L'intestin du Léviathan, aux égouts ou au tas d'ordure: «L'égout, c'est la conscience de la ville.» Ou encore: «Le tas d'ordure a cela pour lui qu'il n'est pas menteur. » Lacan, quant à lui, déclarait:

«Pour ce qui est de reconnaître le passage, le pas, la marque, la trace, la paume de l'homme, nous pouvons être tranquilles - là où nous trouvons une accumulation titanesque d'écailles d'huîtres, ça ne peut manifestement être que des hommes qui sont passés par là. Là où il y a une accumulation de déchets en désordre, il y a de l'homme. [...] Le tas d'ordure - voilà une des faces qu'il conviendrait de ne pas méconnaître de la dimension humaine. » (Lacan 1986: 273-274).

Mentionnons aussi Jean Gouhier fondateur, dans les années 1980, de la rudologie qu’il définit comme la science des déchets des biens et des espaces déclassés: «L'examen des marges renseigne sur le sens, les rythmes, les bases des déclassements et des reclassements. » (1999: 80). L'intérêt scientifique des archéologues pour les déchets est également souligné à maintes reprises. Citons à ce propos Colette Pétonnet, formée par André Leroi-Gourhan:

«Des chasseurs du paléolithique supérieur, on ne saurait rien quant à leur art de vivre si l'on n’avait trouvé qu'un outillage de silex dont l'emmanchement est perdu. Mais des débris de lames autour de l'enclume, les cendres du foyer compactées à une portée de bras vers l'avant, et les os des gibiers tranchés pour en extraire la moelle, rejetés en arrière, ont permis aux préhistoriens de reconstituer le nombre, la disposition, la taille et la forme - circulaire - des tentes. Alors que les outils n'indiquent qu'un certain degré de civilisation matérielle, seules, comme une ironie du temps, les poubelles des Magdaléniens livrent un début de lecture de leur organisation. » (Pétonnet 1991: 108). 
Amas coquiller. Restes d'une activité informelle. Les pêcheurs de moules sur la bande côtière de Temara (Maroc), juin 2016.

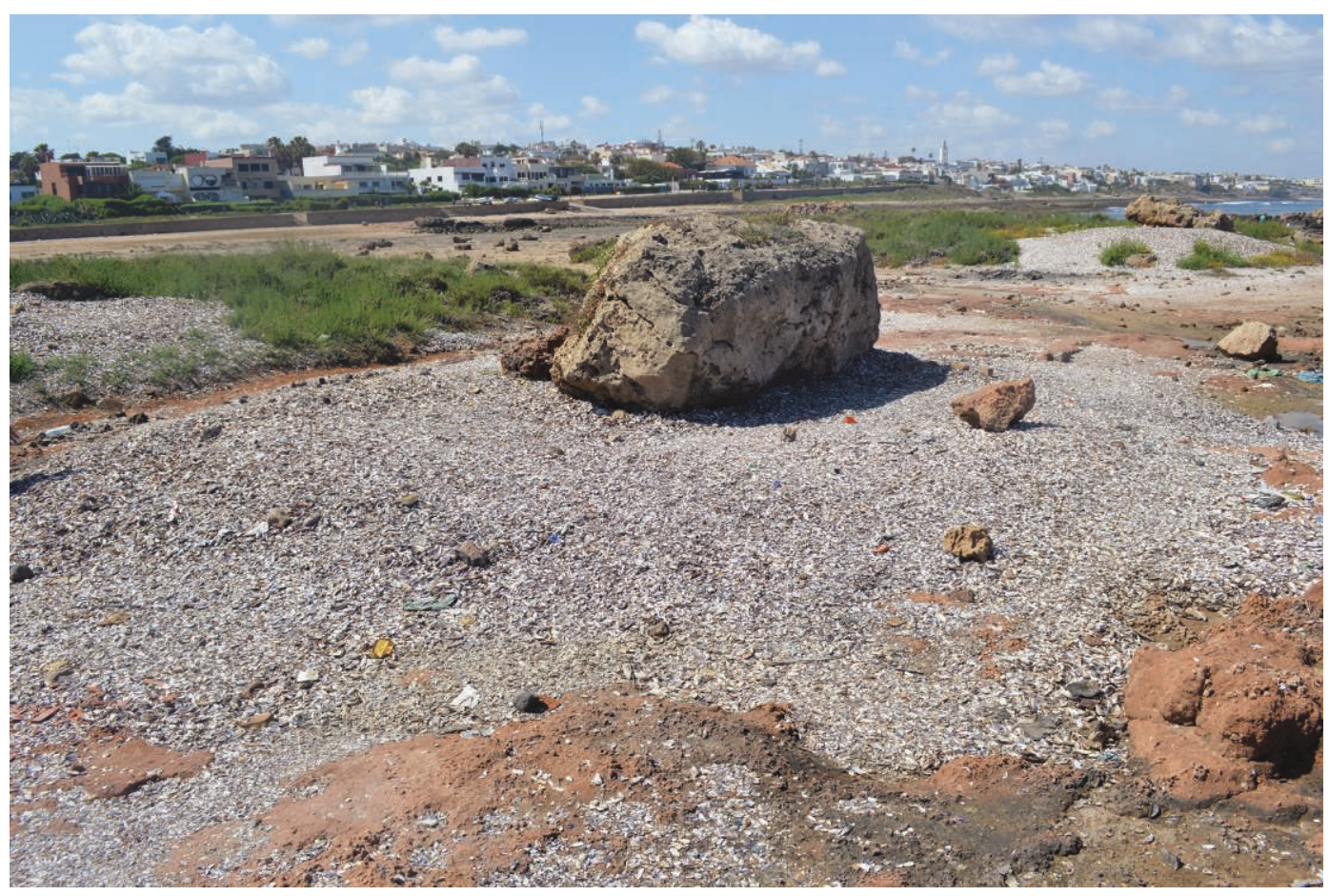

Et l'on ne peut que penser au programme d'archéologie des déchets « Garbage » mis en place en 1973 aux États-Unis par William Rathje et Cullen Murphy. Ce programme s'est déroulé en collaboration avec les employés du service urbain d'assainissement de la ville de Tucson. Les éboueurs étaient désireux d'améliorer leurs conditions de travail et les chercheurs souhaitaient examiner le contenu des poubelles afin d'en retirer des informations relatives aux modes de consommation, aux pratiques alimentaires et sexuelles, plus généralement à la culture matérielle. Les poubelles étaient envisagées comme ne mentant pas. Les chercheurs considéraient que ce qu'ils y trouvaient permettait notamment de rectifier les données obtenues sur la base d'entretiens auprès des habitants. Les responsables du projet soulignent le caractère exotique de ce qu'ils qualifient de «monde des ordures»: "The garbage itself was an unknown world - everything learned about it was new - and thus held the fascination that a trip up the Congo in the nineteenth century would have.» (Rathje \& Murphy 2001 [1992] : 59). Ils relatent ainsi leur rencontre avec le directeur de ce service urbain:

«When representatives of the University of Arizona's anthropology department met with him to discuss their plans to mount a study of Tucson's garbage, and to ask for his help, they found not the hidebound bureaucrat they feared -rigid, myopic, obstructionist-but rather an enlightened despot, a philosopher-garbageman.» (Ibid. : 54).

Ces points de vue, qui accordent aux déchets une dimension heuristique, rejoignent ce que les travailleurs concernés et plus particulièrement les égoutiers - auprès desquels je mène 
depuis 1992 des recherches ethnologiques - disent de leurs activités professionnelles: «Les égouts c'est la vérité», "on développe un savoir psychologique et sociologique sur la vie des gens», «la merde ça ne triche pas».

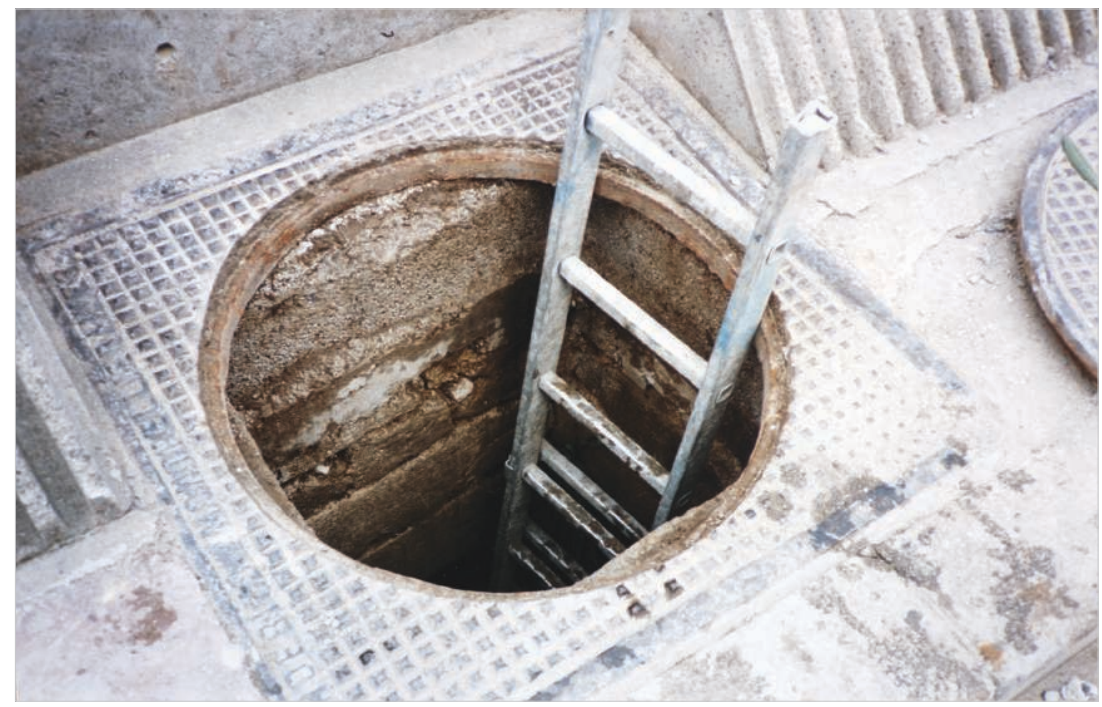

2. «Les égouts, c'est la vérité. »

\section{Mais embourbent ceux qui s'en approchent}

Cette vision nécessite l'adoption d'une posture dont les effets rejaillissent sur celui (chercheur, travailleur des déchets) qui la met en œuvre. Ici encore les points de vue se rejoignent parfois de façon troublante. Ainsi Rathje et Murphy décrivent-ils l'engagement physique des chercheurs dans les mêmes termes, mot pour mot (bien qu'en anglais), que les égoutiers: "Garbarge is not mathematics. To understand garbage you have to touch it, to feel it, to sort it, to smell it [...]» (Rathje \& Murphy 2001 [1992] : 9).

Sur mes terrains de l'assainissement, j'ai entendu maintes fois cette phrase: «La merde, pour comprendre il faut toucher» (des égoutiers in Jeanjean 1998). Si tous les chercheurs ne « touchent pas» toujours, ils sont toutefois atteints par les objets auxquels ils s'intéressent. Usant de la métaphore, François Dagognet évoque la menace d'un «embourbement » (Dagognet 1997 : 11). Freud écrivait voici un siècle:

«Il suffira de mentionner une seule de ces conséquences, celle qui, ici, nous touche le plus: il n'a pas été permis à la science de s'occuper de ces aspects proscrits de la vie humaine, en sorte que quiconque étudie de telles choses se voit considéré comme à peine moins "inconvenant" que celui qui fait réellement des choses inconvenantes. » (Freud 1981 [1913] : 33). 
Il fait ici allusion au travail de Bourke sur les rites scatologiques (Bourke 1981 [1891]). En 1999, dans un ouvrage consacré aux déchets, Yann Cochin et Dominique Lhuilier reprenaient cette idée et évoquaient la difficulté pour le chercheur de se confronter au regard de ses pairs, «regard au pire méprisant, au mieux compatissant sur cet étrange intérêt » (Lhuilier \& Cochin 1999: 15). Bien qu'il existe encore des objets de recherche nettement plus valorisants et valorisés, cette dimension tend à s'estomper.

Les déchets sont dans l'air du temps. L'engouement - dans la société française - pour les vide-greniers, les ressourceries et autres dispositifs qui se proposent de donner une deuxième vie aux objets en témoigne. Leur prolifération intimement articulée à la valorisation de la « croissance» soulève des inquiétudes mais également des enjeux économiques considérables et ils sont l'objet de multiples attentions - y compris dans le champ artistique.

Du côté des sciences sociales, depuis les années 2010, le nombre de travaux et de publications qui leur sont consacrés croît de façon exponentielle. Si les chercheurs n'ont plus à batailler pour défendre l'intérêt et la légitimité de leurs objets de recherche, il devient en revanche difficile dans nos écrits, de plus en plus nombreux, d'éviter les redondances. Les usages métaphoriques des termes relatifs aux déchets compliquent la tâche. En effet, il s'agit ici de tenir une position singulière dans la mesure où nous travaillons sur des substances, des états dont l'expression métaphorique ${ }^{1}$ est extrêmement utilisée, y compris dans nos disciplines, pour qualifier des individus et exprimer des situations sociales. Cela a-t-il du sens de parler de déchets ou de rebuts lorsqu'il s'agit d'êtres humains ${ }^{2}$ ? De cadavres? Où se situe la limite entre naturalisation, procédé rhétorique heuristique, effet de style? Comme le souligne Baptiste Monsaingeon dans sa thèse de doctorat (Monsaingeon 2014), ces questions se compliquent d'autant plus que les problématiques relatives aux déchets et aux restes s'inscrivent dans un champ en vogue, économiquement porteur et prolixe en oxymores et autres contradictions, celui du « développement durable».

Les travailleurs évoquent des difficultés semblables. Et plus particulièrement lorsqu'ils ont affaire à des objets, substances ou états de la matière qui inspirent le dégoût - des déchets organiques notamment. Ils sont confrontés aux usages métaphoriques des termes relatifs aux déchets qui rendent difficile leur emploi au sens propre. Ils font face aux euphémismes et autres figures rhétoriques conduisant à occulter, à dénier le réel de leur activité. Ils expliquent ne pas trouver les mots pour dire à ceux qui n'exercent pas leur métier «la merde qui pue, qui colle et qui coule» (des égoutiers in Jeanjean 1998). Il suffit ici de prêter l'oreille pour constater l'ironie de la situation et entrevoir toutes les difficultés d'une entreprise qui consiste à concevoir un sens propre pour dire le sale. Les mots, et plus particulièrement leurs usages socialement admis, semblent n'avoir que très peu de prise sur la matière, leur efficacité est régulièrement mise en doute ${ }^{3}$ : «On a beau écrire sur nos camions le mot propreté ça reste des pompes à merde et nous on est dedans [...] Je suis quelqu'un qui se fait chier dessus, au sens propre comme au figuré.» (Ibid.) ${ }^{4}$. 


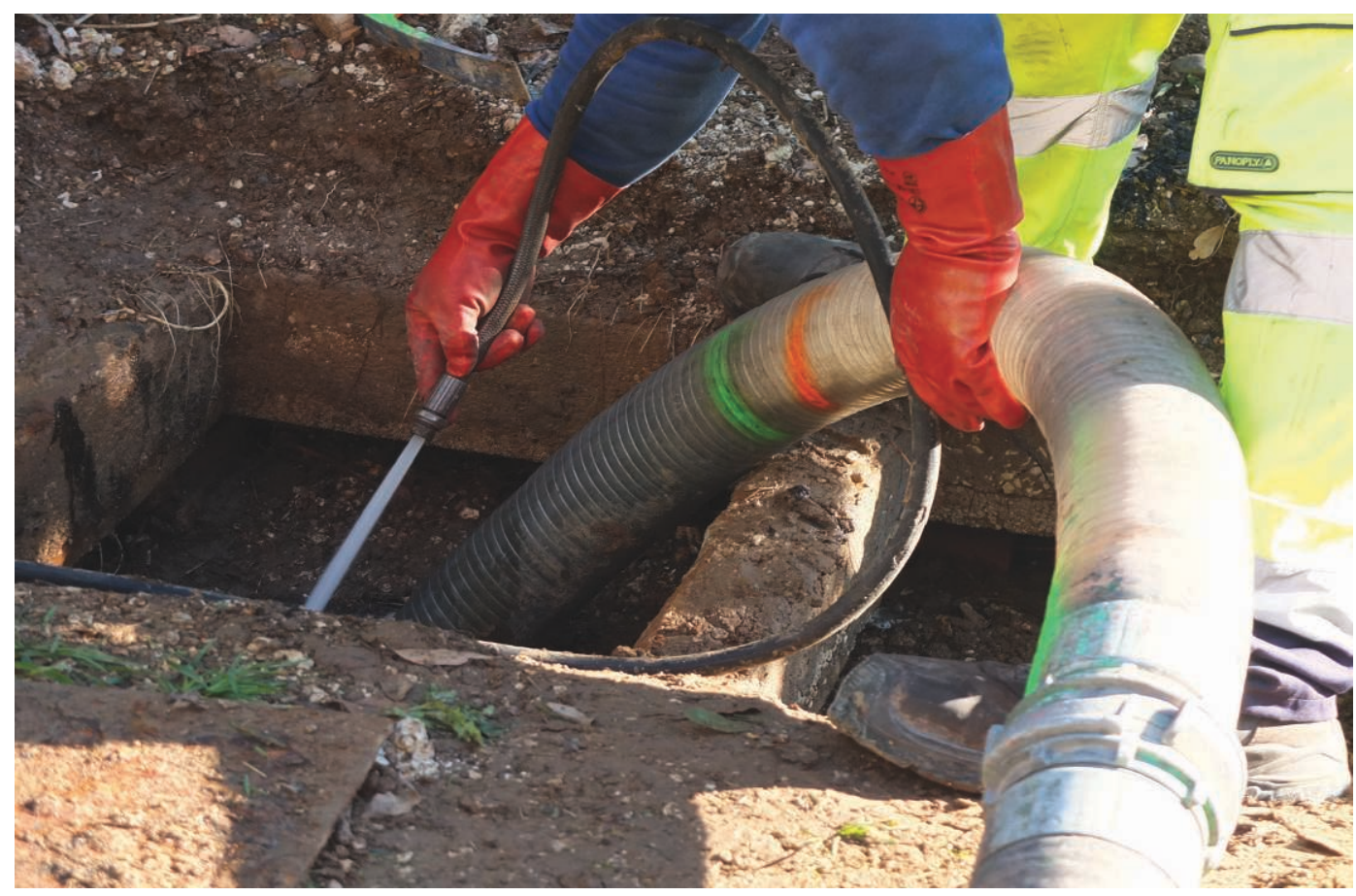

3. Face à face avec la matière.

\section{Là où le sens vacille}

Auprès des travailleurs en contact avec des déchets ou des restes humains (égoutiers, agents de chambres mortuaires,...), des questions relatives au sens de leurs vies, à leur équilibre psychologique, à la dimension hautement signifiante de leurs gestes sont présentes à chaque pas. Les travailleurs font part de moments où l'angoisse surgit et le sens vacille (Jeanjean $\&$ Laudanski 2014). Là encore nous rejoignons certains auteurs, Kristeva, Bataille, ou encore de Certeau pour lequel «tout ordre autonome se constitue grâce à ce qu'il élimine et il produit un reste condamné à l'oubli, mais l'exclu s'insinue de nouveau dans cette place "propre", il y remonte, il l'inquiète [...] » (Certeau 1987 : 86). L'abjection, les objets chus, la «part maudite» ont à voir avec le sens. Ici l'ethnographie rejoint la psychanalyse. Robert Samacher, dans son ouvrage sur la pulsion de mort, écrit:

«Ainsi, ma génération, celle qui a été bercée dans le yiddish mameloshn, est encore en mesure de retransmettre l'esprit du yiddish et de la yiddishkeyt dans les débris, les déchets ordinaires de la réflexion par lesquels circulent la métaphore et la métonymie et qui sont des parcelles signifiantes impliquées dans la création de sens. » (Samacher 2009: 61). 
À la suite de Lacan, Samacher articule le reste déchu au désir et l'inscrit parmi les objets a qu'il pense comme «un reste de jouissance qui se détache du corps [...] et qui en tant qu'objet cause du désir ne cesse de nous faire courir (Samacher 2009: 25-26). Ici sens et désir se lient. Souvenons-nous dès lors des pages de Lévi-Strauss à propos du bricoleur:

«Tous ces objets hétéroclites qui constituent son trésor, il les interroge pour comprendre ce que chacun pourrait "signifier", contribuant ainsi à définir un ensemble à réaliser, mais qui ne différera finalement de l'ensemble instrumental que par la disposition interne des parties [...] il "parle", non seulement avec les choses, comme nous l'avons déjà montré, mais aussi au moyen des choses: racontant, par les choix qu'il y opère entre des possibles limités, le caractère et la vie de son auteur. » (Lévi-Strauss 1983 [1962] : 32-35).

4. Mobylette et moules Les dessous de la ville à ciel ouvert. Nettoyage du canal Saint-Martin, Paris 2016.

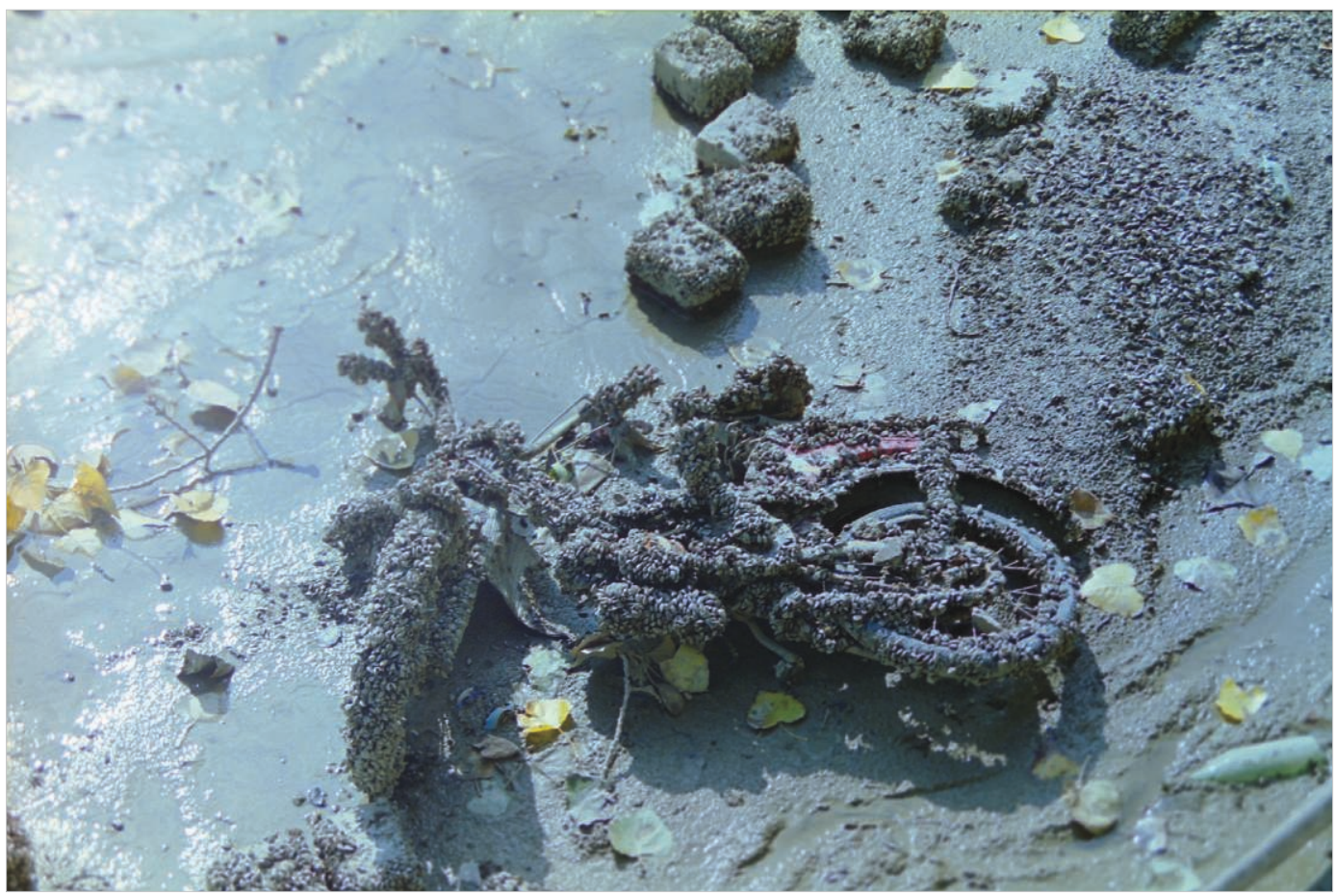

Ou encore Marcel Mauss qui, dans son texte «les techniques du corps», fouille un tas de restes abandonné par ses collègues universitaires sous l'étiquette «divers». Mauss nous enseigne alors que la «libido sciendi réside dans ces restes inexplorés, comme autant de territoires vacants propices au développement et à l'exercice de la pensée ». (Mauss 1993 [1950]). Cela fait encore écho à la réflexion d'égoutiers lorsque ceux-ci puisent matière à penser dans l'opacité des eaux usées et le trouble qu'elles suscitent. Ils opposent, par exemple, 
les eaux d'égouts aux eaux propres (potables) considérant que ces dernières, «transparentes, insipides, sont ennuyeuses et n'ont rien à dire », alors que les eaux usées sont à leurs yeux «épaisses, troubles, et de ce fait intéressantes » (des égoutiers in Jeanjean 1998). L'épaisseur ainsi évoquée est physique, puisque l'eau charrie des objets, des matières en suspension. Mais il s'agit aussi de l'opacité des raisonnements qui s'appliquent aux eaux usées, des interdits, des hontes et des secrets qui les environnent. "On découvre toujours des choses, des histoires, des fonctionnements psychologiques", aime dire un chef de service (aujourd'hui retraité), qui considère par ailleurs que «la pureté des eaux potables les rend inintéressantes et d'une grande platitude». Ainsi articule-t-il à son activité professionnelle une position philosophique quant à la "pureté». Ce point de vue était partagé par une majorité de ses collègues de travail, agents municipaux à présent retraités ou décédés.

\section{Et l'écriture peine}

Ces travailleurs considèrent par ailleurs que leur domaine d'activité, troublant, épais de sens, riche de vérités et de savoirs secrets, oppose des résistances à sa mise en écriture. Dans les années 1990, les égoutiers montpelliérains me répétaient inlassablement: «Les égouts ça ne peut pas s'écrire», «l'écriture dans les égouts ce n'est pas la vérité», ou encore «si je savais écrire, j'écrirais des livres et des livres, j'écrirais partout sur les pompes à merde ». Nous pouvons voir là une protection classique articulée à la valorisation de savoirs oraux et aux formes de transmission qui les accompagnent, une résistance aux écrits prescrits et l'entretien d'une opacité protectrice vectrice de liberté... Autant de mécanismes observés ailleurs par des sociologues ou ethnologues du travail. Mais nous pouvons aussi, et dans le même temps, y voir un questionnement quant à la possibilité d'écrire le déchet, plus particulièrement les excréments et les déchets organiques. Les chercheurs se confrontent à cette difficulté. Nous avons évoqué plus haut quelques-unes des questions qui se présentent dès lors qu'il s'agit de dire l'excrément, le rebut, le déchet. Dominique Lhuilier et Yann Cochin soulignent à ce propos qu'« ici, comme toujours mais plus que jamais, il appartient au signe comme tel d'exercer une fonction de déni à l'endroit du réel qu'il signifie» (Lhuilier \& Cochin 1999: 88). S'appuyant notamment sur la réflexion de Julia Kristeva, ils mettent en exergue l'irréductibilité du réel qui «ne se laisse pas prendre dans les ressorts du symbolique» (ibid.: 97). Le psychanalyste Dominique Laporte a travaillé de façon très fine cette articulation entre écriture et déchets, et plus particulièrement entre expérience littéraire et scatologie. Il insiste sur l'ambivalence des déchets. Le mouvement d'oscillation entre bons et mauvais objets est mis en lumière par de nombreux auteurs, non seulement des psychanalystes, mais également des économistes (Bertolini 1978, Latouche 1972) et des philosophes (Dagognet 1997, Harpett 1999). Ainsi Aurel Kolnaï écrit-il à propos du dégoût très présent aux abords des objets dont nous traitons ici : «La "provocation" qui émane du dégoûtant a une double signification - conformément 
à l'ambivalence [...] Elle est à la fois invitation et dissuasion, appel et menace [...]» (Kolnaï 1997 [1929] : 84). Dans son texte introductif à la réédition de l'ouvrage de Bourke, Les rites scatologiques, Laporte écrit: "Ce que la scatologie partage avec l'expérience littéraire c'est, à n'en pas douter, cette mitoyenneté où le Tout se révèle strictement contigu au Rien. »C'est en ce point qu'il situe l'écrit de Bourke: «On n'écrit pas plus la merde qu'on ne peint la voix. Si le livre de Bourke a un objet, il faut peut-être le tenir pour défini par cet impossible même, par la limite où l'écriture est en mesure de basculer du tout au rien.» (Laporte 1981 : 21). Ici les réflexions des égoutiers à propos d'une écriture tout à la fois impossible, fallacieuse et souhaitée prennent toute leur profondeur. Il arrive toutefois que l'écriture ethnographique satisfasse leurs attentes. J'ai pu le constater auprès d'égoutiers montpelliérains, et des collègues sociologues ont établi le même constat lorsqu'ils ont diffusé leurs publications sur leurs terrains d'enquête ${ }^{5}$. L'écriture ethnologique fait sens pour les travailleurs parce qu'elle a une dimension littéraire, mais aussi parce qu'elle restitue ce que font disparaître les écrits prescrits ainsi que tous les documents soucieux de livrer une image «présentable » des déchets et des activités qui s'y rattachent. Les «travailleurs des déchets» (Corteel \& Le Lay 2011) et les chercheurs en sciences humaines n'occupent pas les mêmes places vis-à-vis des rebuts, pas plus qu'ils ne partagent les mêmes positions sociales. Toutefois les uns et les autres sont confrontés

Retranscription à la surface du cheminement souterrain des égouts.

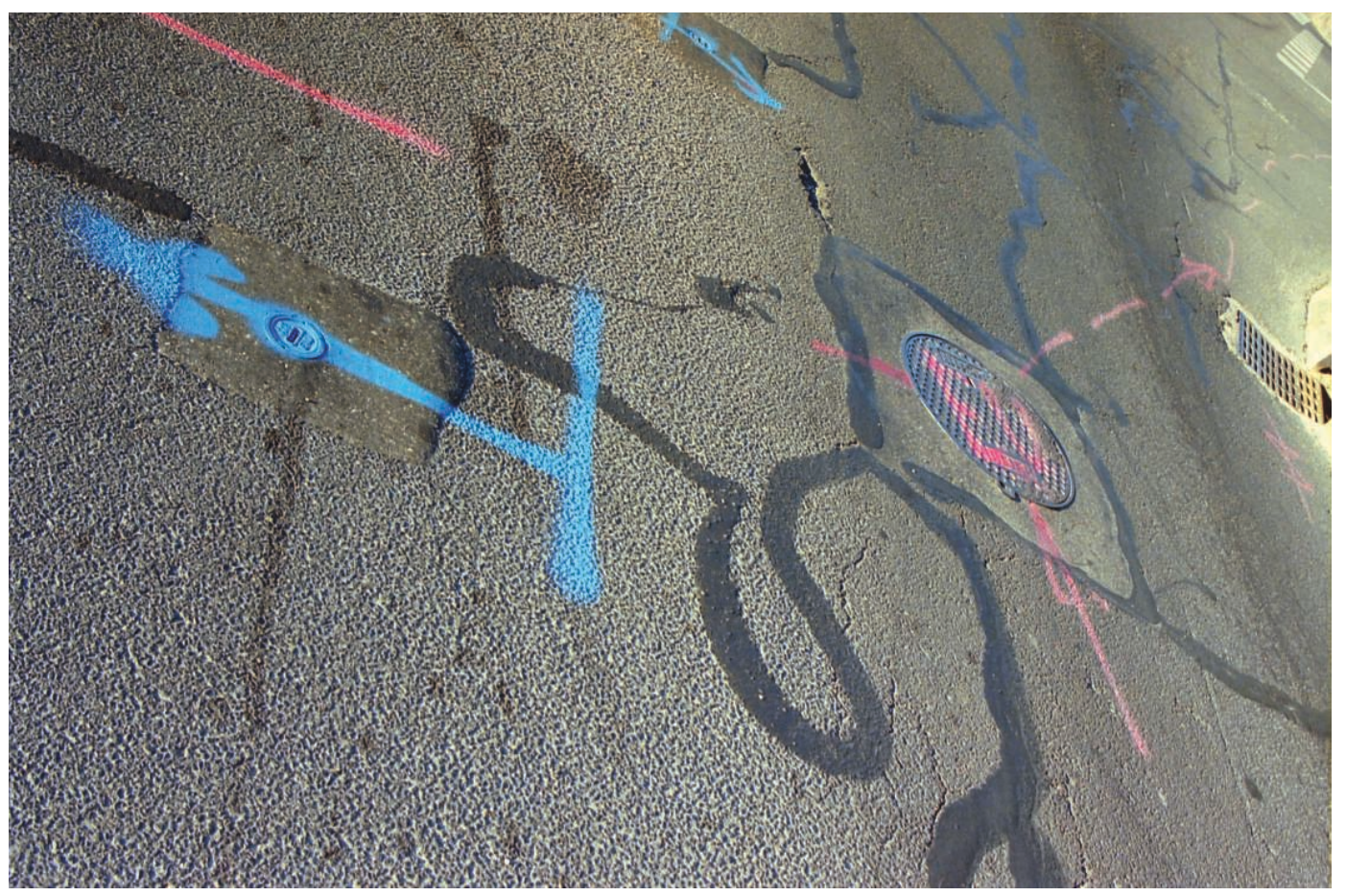


à ces états de la matière et à leurs effets symboliques. Il est des expériences et des questions communes. Parfois les solutions des uns apportent des éléments de réponse aux autres.

Pour conclure cet article, et en m'appuyant sur les mises en perspective que j'y ai engagées, je me permets de soumettre au lecteur un point de vue plus «général» à propos des déchets en tant qu'objet de recherche pour les sciences humaines et sociales. Au fil de cette réflexion, je me suis essentiellement appuyée sur des paroles d'égoutiers au contact d'eaux usées et d'excréments humains. Ces derniers constituent des déchets bien particuliers dans la mesure où ce sont des déchets humains organiques. Il ne s'agit pas d'ordures ménagères, d'objets manufacturés, pas plus que de substances radioactives ou de « déchets ultimes ». Les eaux usées et les excréments renvoient directement aux façons dont les sociétés articulent les corps biologiques (mais aussi dans leur dimension érotique, affectif,...) et le corps social, à la place socialement accordée aux fonctions naturelles. Les historiens ont montré combien les dispositifs techniques qui s'y appliquent, tout au moins en Occident, sont les produits de processus historiques de refoulement, de mise au secret des fonctions naturelles et des plaisirs qui leur sont attachés. Ces processus de refoulement s'appuient sur des affects tels la honte, l'angoisse, le dégoût. Sans perdre de vue les caractéristiques de ces substances, des techniques et positions sociales qui s'y articulent, je considère, à la lumière de travaux comparatifs auprès d'agents de chambres mortuaires (Jeanjean \& Laudanski 2014) et d'éboueurs (Corteel \& Le Lay 2011), que les eaux usées, les excréments humains et les représentations qui les environnent peuvent nous aider à penser d'autres restes, que ceux-ci soient déchus ou non. Pour cela, nous ne devons pas céder aux généralisations rapides, mais prendre en compte les différences et toutes les subtilités qui en découlent. Nous ne pouvons penser tous les restes, et en particulier les déchets, de la même façon, mais nous pouvons les penser les uns à l'aune des autres en croisant les regards disciplinaires. Se pose alors la question de l'existence d'un champ de recherche. La multiplication récente, en sciences humaines et sociales, de travaux sur les déchets est très évidemment liée à l'intérêt que leur portent les différents acteurs du marché concurrentiel; mais aussi aux orientations politiques et économiques contemporaines. La croissance en termes de production et de consommation génère inévitablement une présence accrue de déchets. Beaucoup de recherches sont le produit de commandes et appels d'offres publics ou privés souvent articulés à l'idéologie du développement durable. En France, l'ADEME ${ }^{6}$ par exemple a joué un rôle important, un grand nombre de travaux ayant été impulsés et financés par cet organisme public. Il serait intéressant, et même indispensable, afin de constituer un véritable « champ de recherche» que les chercheurs prennent de la distance vis-à-vis de ces orientations. Non pas seulement en adoptant un point de vue critique vis-à-vis des organismes financeurs ${ }^{7}$, mais en prenant le temps d'instaurer des décalages entre de nouvelles questions de recherche et celles construites à partir de ces premières orientations. Ce processus est manifestement en cours. 


\section{Notes}

1. À propos de ce mécanisme, voir par exemple Lou Andreas-Salomé 1915.

2. Zygmunt Bauman utilise les termes «déchets humains», « rebuts humains», «êtres humains rejetés» (Bauman 2009 [2004] : 19).

3. Bien évidemment, ceci n'est pas spécifique aux déchets mais contenu dans le langage même. Toutefois, ce questionnement est particulièrement présent sur les terrains de l'assainissement, il fait partie des difficultés du métier et revient de façon extrêmement récurrente dans les discussions et entretiens.

4. Georges Knaebel considère que l'excrément «ne devient exprimable qu'au prix de sa dépréciation, de sa dérision, de son déni » (Knaebel 1991).
5. C'est le cas par exemple de Stéphane Le Lay que je remercie d'avoir bien voulu partager avec moi son expérience de sociologue auprès des éboueurs de la ville de Paris.

6. Agence de l'environnement et de la maîtrise d'énergie.

7. S'en tenir à ce préalable indispensable garantit, entre autres, la bonne conscience des chercheurs, mais ne permet pas forcément la diversification des questions de recherches. Nous observons plutôt une uniformisation des points de vue et des discours critiques.

\section{I'auteure}

Agnès Jeanjean est ethnologue, maîtresse de conférences en ethnologie à l'université de Nice Sophia-Antipolis. Membre du Laboratoire d'anthropologie et de psychologie sociales et cognitives, elle propose une anthropologie de la société par ses restes. Ses travaux portent principalement sur les travailleurs des déchets, les habitats de fortune et les transformations contemporaines de l'organisation du travail.

\section{Iconographie}

Image d'ouverture. Travailleurs des égouts, Rabat, 2001. (C) Agnès Jeanjean

1. C) Émilie Campmas

2 et 4. (C) Agnès Jeanjean

3. (C) F. Joulian

5. (C) P. Guigou

\section{Références}

Andreas-Salomé, L. 1980 [1915] «Anal et sexuel » in L'amour du narcissisme. Textes psychanalytiques. Paris: Gallimard: 91-130.

Bauman, Z. 2009 [2004] Vies perdues. La modernité et ses exclus. Paris: Payot.

Bertolini, G, 1978 Rebuts ou ressources? La socio-économie du déchet. Paris: Éditions Entente.

Bourke, J.G. 1981 [1891] Les rites scatologiques. Paris: PUF.

Certeau, M. de 1987 Histoire et psychanalyse entre science et fiction. Paris: Gallimard.

Corteel, D. \& S. Le Lay dir. 2011 Les travailleurs des déchets. Toulouse: Érès.

Dagognet, F. 1997 Des détritus, des déchets, de l'abject. Une philosophie écologique. Paris: Les empêcheurs de tourner en rond.

Freud, S. 1981 [1913] «Préface» in J.G.. Bourke Les rites scatologiques. Paris: PUF.

Gouhier, J. 1999 « La marge entre rejet et intégration » in J.-C. Beaune Le déchet, le rebut, le rien. Paris: Champ Vallon.

Harpett, C. 1999 Du déchet. Philosophie des immondices. Paris: L'Harmattan.

Hugo, V. 1862 Les Misérables. L’intestin de léviathan. Tome5, livre2, chapitre 2. 
Jeanjean, A. 1998 Basses Euvres. Approche ethnologique d'un réseau technique urbain: les égouts de Montpellier. Thèse de doctorat, Université Paris V.

Jeanjean, A. \& C. Laudanski 2014 «Comment y "mettre les mains". Les travailleurs du funéraire face à la manipulation des corps morts», Techniques \& Culture 60: 172-187.

Knaebel, G. 1991 «Le rangement du résidu », Les Annales de la recherche urbaine 53: 23-31.

Kolnaï A. 1997 [1929] Le dégoût. Paris: Agalma.

Lacan, J. 1986 Le séminaire livre VII. Paris: Seuil.

Laporte, D.G. 1981 «Présentation» in J.G. Bourke Les rites scatologiques. Paris: PUF.

Latouche, S. 1972 «Le revers de la production: éléments pour une approche nouvelle des mythes et des réalités de la pollution», Traverse 12 (2): 85-100.
Lévi-Strauss, C. 1983 [1962] La pensée sauvage. Paris: Plon. Lhuilier, D. \& Y. Cochin 1999 Des déchets et des hommes. Paris: Desclée de Brouwer.

Mauss, M. 1993 [1950] Sociologie et anthropologie. Paris: PUF.

Monsaingeon, B. 2014 Le déchet durable. Éléments pour une socio-anthropologie du déchet ménager. Thèse de doctorat, Paris 1 Panthéon-Sorbonne.

Pétonnet, C. 1991 «Le cercle de l'immondice», Les Annales de la recherche urbaine 53: 108-111.

Rathje, W. \& C. Murphy 2001 [1992] Rubbish! The Archaeology of Garbage. New york: Harper Collins Publishers.

Samacher, R. 2009 Sur la pulsion de mort. Création et destruction au coeur de l'humain. Paris: Herman.

\section{Pour citer cet article}

Jeanjean, A. 2016 «Peindre la voix, écrire le déchet», TechniquesECulture 65-66 «Réparer le monde. Excès, reste et innovation», p. 310-321. 